Meta

Journal des traducteurs

Translators' Journal

\title{
Phraséologie et terminologie en traduction et interprétation
}

\section{Yves Gambier}

Volume 38, numéro 2, juin 1993

URI : https://id.erudit.org/iderudit/002796ar

DOI : https://doi.org/10.7202/002796ar

Aller au sommaire du numéro

Éditeur(s)

Les Presses de l'Université de Montréal

ISSN

0026-0452 (imprimé)

1492-1421 (numérique)

Découvrir la revue

Citer cette note

Gambier, Y. (1993). Phraséologie et terminologie en traduction et interprétation. Meta, 38(2), 389-391. https://doi.org/10.7202/002796ar d'utilisation que vous pouvez consulter en ligne.

https://apropos.erudit.org/fr/usagers/politique-dutilisation/ 


\section{PHRASÉOLOGIE ET TERMINOLOGIE EN TRADUCTION ET INTERPRÉTATION}

Sept cents ans pour la Confédération helvétique ; cinquante ans pour l'École de traduction et d'interprétation de Genève... Voilà deux anniversaires marquant la stabilité, la continuité - alors même que 1991 aura connu des transformations rapides, ici et là, en Europe notamment. C'est dans ce paysage contrasté qu'a eu lieu les 2,3 et 4 octobre 1991 un colloque international, «Phraséologie et terminologie en traduction et en interprétation», excellemment organisé par l'ETI - avec interprétation simultanée en français, anglais et allemand - dans les locaux de la Faculté de médecine.

Les objectifs déclarés de la rencontre étaient clairs: faire avancer la recherche sur les collocations / expressions et locutions, dans leur rapport à la traduction, à l'interprétation, aux langues de spécialité, à la terminologie, à la lexicographie. Cette avancée devait à la fois couvrir la réflexion théorique et les solutions pratiques.

Le lieu, le thème ont amplement justifié la dimension internationale du colloque: plus de 350 participants d'une trentaine de pays ont ainsi accepté d'écouter près d'une cinquantaine d'exposés. Il y avait longtemps que la terminologie n'avait pas fait un tel plein.

Douze séances ont eu lieu en parallèle, entourées par deux matinées de plénières. Il est bien sûr impossible de rendre compte de l'ensemble. Les remarques qui suivent sont donc d'ordre général et partielles: tous les orateurs ne peuvent être cités et certains pourraient l'être sous plus d'une remarque. L'agencement et le découpage des sous-thèmes sont souvent épineux. On peut trouver sans doute bien des raisons a l'ordre finalement retenu à Genève - lui-même fonction de la quantité des contributions acceptées. Mais ne dit-on pas : qui trop embrasse mal étreint... En quoi ainsi la traduction littéraire (4 exposés), même rattachée aux questions de l'idiomaticité, devait-elle avoir la priorité sur la problématique même du col- 
loque, traitée dans les deux derniers ateliers (6 exposés)?

En quoi les expériences en traduction spécialisée devaient-elles prendre le pas sur les interrogations touchant la matière même des idiomes, des collocations (exposés de M. Cl. L'Homme, J. Moeschler, W. Martin, H. Béjoint, U. Reid...)? Questions d'autant plus cruciales qu'au cours de ces trois jours, la terminologie uni- et multilingue des terminologues, des linguistes, des traducteurs est apparue flottante, mal assurée - comme en octobre 1986, à Paris, sur la «fertilisation terminologique dans les langues romanes» - à propos des emprunts, des calques hybrides / partiels, etc.

Ont été en effet traités les idiomes, les clichés. les dictons, les maximes, les métaphores, les expressions toutes faites ou consacrées, les slogans, les formules concises... (exposés de JP. Neuhaus, I. Hamilton, J. Munoz, M. Voisin...). Par ailleurs, phraséologie et syntagme ont eu tendance à se mêler confusion fâcheuse quand on sait déjà que les amalgames, la polysémie, n'aident guère à clarifier le débat linguistique depuis Bally, Saussure, Firth, Martinet jusqu'à Guilbert, Kokourek, Mel'čuk (exposés de R. Goffin, G. Budin, G. Gréciano...). Des définitions opératoires, temporaires - à défaut d'être consensuelles $-\mathrm{n}$ 'auraient-elles pas permis certains approfondissements? L'effort définitoire implique-t-il le délire théorique?

La présence de nombreux praticiens aurait pu apporter des réponses intéressantes. Mais - et c'est la deuxième remarque - ils ont préféré s'en tenir à un empirisme artisanal: on fait de la phraséologie «appliquée», sans se préoccuper de cerner l'objet dont on parle! Cet aveuglement n'est pas nouveau et A. Rey, dans sa synthèse, a dû encore repréciser les rapports entre théorie et pratique, la nécessité de dépasser des positions par trop dogmatiques. Peut-on rechercher une reconnaissance des spécificités des métiers langagiers sans s'en donner les moyens, c'est-à-dire sans se mettre à distance de ses tâches, pour ne plus confondre recettes et réflexions? Cette fixation sur la répétition du quotidien, ce refus de poser les problèmes autrement qu'en termes utilitaristes à courte vue... ont fait que la phraséologie a été un peu l'Arlésienne: on en a parlé certes sans la percevoir beaucoup... Fallait-il recouvrir encore les dilemmes, les incertitudes de cette phraséologie déjà (mal) traitée par les linguistes et redécouverte par plusieurs autres colloques récents (Mannheim (1981), Zurich (1984), Oulu (1986), Strasbourg (1988), Uppsala (1990)) ? D'une certaine manière, les interprètes ont caricaturé cette position, la phraséologie n'étant qu'un prétexte pour aborder certains points propres à leur métier - ce qui n'enlève pas la pertinence de ces points ni la qualité des exposés...

La phraséologie est-elle une catégorie fourretout, sinon un cache-misère? Pourquoi les formes composites, parfois lexicalisées, parfois répertoriées - pour les locutions, les formules de politesse, de correspondance, la terminologie de conférence... - sont-elles si résistantes au questionnement du praticien et à la pratique du théoricien? En aval des termes complexes et en amont des macrostructures du discours, elles n'offrent pas forcément la stabilité du lexique tout en jouant des moyens de la syntaxe : elles sont des énoncés souvent familiers que notre sentiment de la langue rend acceptables, mais encore irréductibles, semble-t-il, à une analyse systématique, univoque.

Entre l'aveuglement relatif des langagiers et le quasi mutisme des linguistes, que pouvaient troisième remarque - les terminologues?

Reconnaître que les termes se déploient avec des non-termes, selon des contraintes morpho-syntaxiques, rhétoriques, selon certaines relations sémantiques, c'est bousculer la définition stricto sensu du terme vite réduit au substantif, c'est réhabiliter ces motsoutils (verbes, prépositions...) si nombreux dant tout texte et si négligés, sinon exclus, par la terminologie dominante. D'une façon, la phraséologie brouille les frontières entre la langue dite générale et celles dites de spécialité, entre la «langue» et la «parole»: avec elle, on se doit de saisir sur le vif les interrelations entre langue, langage, discours, règles syntaxiques et unités lexicales et terminologiques. En abordant les collocations - combinaisons syntagmatiques plus ou moins figées, le terminologue est contraint d'aborder les mécanismes de signification tels qu'ils sont «bricolés» dans les communications spécialisées. On peut dire que les terminologues ont été étrangement silencieux sur les prolongements (théoriques et méthodologiques) éventuels de cette prise en considération de la phraséologie. Les questions de repérage, d'identification, de découpage, de description, de classification n'ont été que trop succinctement abordés, en particulier par les auteurs préoccupés par le traitement informatique. Ont été négligés la fonction de cette phraséologie dans le texte, son rôle et sa place dans la cohérence textuelle. (voir les exposés de D. Blampain, Cl. Bocquet, H. Brisson, H. Bühler, B. Cohen, F. Gaudin, U. Heid, B. Leblanc, E. Ten Pas, A. Reichling, P. Shann, S. Tagnin, C. Yallop...).

Deux autres questions ont été brièvement analysées mais dans un rapport finalement assez lâche à la phraséologie :

- celle de l'équivalence, plutôt confondue avec l'appariement en «langue» (exposés de M. Hánaková, M. Viezzi...), parfois mêlée au problème de l'apprentissage du décodage/encodage des tournures idiomatiques étrangères (voir JP. Colson, G. Jorge...) et

- celle des variations géographiques (comme dans $\mathbf{M}$. Abdel Hadi, J. Dancette, N. Gimelfarb...), ce qui permet ici de signaler les langues citées en exemples : l'allemand, l'anglais, l'espagnol, le français, l'italien et une ou deux fois le portugais, le grec, le néerlandais, le slovaque.

Les réponses apportées à ces deux questions ont laissé transparaître - et ce sera la quatrième et dernière remarque - un postulat très fort, implicite tout au 
long des trois jours et déjà vaguement formulé dans la première annonce du colloque : la communication interlinguistique doit viser à «l'authenticite»», à «la recherche de l'expression la plus idiomatique» affirmation parfois jusqu'à la caricature au nom d'une certaine lisibilité et d'une certaine "efficacité» (voir H. Bergmann, D. Birraux, H. Eisele, M. Rochard...) La compilation phraséologique ne serait-elle alors qu'une façon d'effacer le travail de transfert? Dans cette perspective de réduire la voix de l'autre aux normes de soi (langue et culture d'arrivée), le traducteur et l'interprète en viennent à n'être que reproducteurs de clichés. Ne traduit-on vraiment que des textes stéréotypés pour des lecteurs stéréotypés? La traduction se réduit-elle à une obsession puriste pour plaire et se complaire dans les formes les plus attendues, les plus conventionnelles du public cible ? On conviendra que ces questions n'étaient pas directement du ressor des participants et pourtant l'exigence d'idiomaticité - pour le traducteur, pour l'apprenant en langues peut conduire à un leurre, à une illusion... que la terminologie n'a pas à entretenir.

Derrière le recours à la phraséologie - inventoriée, informatisée - peuvent s'esquisser le rêve d'une langue de bois (c'est d'ailleurs un des sens de «phraséologie»: usage de phrases creuses), le désir un peu fétichiste d'incorporer les manières de dire de l'autre - «comme si» on était à sa place, comme si la traduction n'était pas une traduction, comme si on était natif d'une langue étrangère. Vertige des dimensions anthropologique et sociale de ces prises de position...

Par ailleurs, peut-on réduire les langues à de tels stocks stables, à de tels conservatoires de formes habituelles, alors que la parole introduit sans cesse des glissements de sens, des ruptures créatrices? La tentation positiviste de survaloriser le seul fonctionnement dénotatif du langage, d'expulser les phénomènes de ratages existe. Une terminologie qui confondrait phraséologie et «répertoire immobile» (Benveniste) de formes figées, qui oublierait «l'activité métamorphique» (id.) de la langue serait vite interpellée par les usages pluriels. Ici les traducteurs littéraires (voir l'exposé de M. Legros-Chapuis) auraient pu démontrer comment le langage porte en lui-même sa propre subversion; faut-il rappeler les acquis et les réflexions de Breton, de Desnos, de Vian, de Joyce, d'Artaud, du lettrisme.... les jeux du surréalisme, des publicitaires, les détournements des slogans politiques... et autres calembours qui permettent les références, les allusions à une certaine sagesse accumulée dans l'histoire, les clins d'œil, la complicité dans l'échange intraculturel... La phraséologie est à la fois cette tendance et cette tentation à enfermer la langue, à en suspendre les innovations ET un des lieux, un des ressorts possibles de sa créativité, de la réalisation de ses potentialités. Langue et culture sont trop souvent perçues et apprises normativement. Or dans les interactions quotidiennes, dans l'échange des formes littéraires... on fait face à nombre de situations non normatives. La recherche en phraséologie ne saurait masquer la nécessité de cette flexibilité : la langue, le discours ne sont pas des catégories hypostasiées, la traduction n'est pas l'application répétitive, rigide de normes, de conventions établies une fois pour toutes.

Pendant ces trois jours, on a plaidé plus ou moins ouvertement pour une approche plus discursive de la terminologie; on a ignoré les lacunes, les manques de la linguistique générale pour traiter des énoncés infraphrastiques; on a refoulé la dialectique entre théorie et pratique; on a touché aux critères variés. mixtes qui pourraient circonscrire les traits distinctifs de l'idiomaticité ; on a négligé la part et le fonctionnement de la phraséologie dans l'oral (spontané, savant)... Une certaine tension, sinon une contradiction, s'est fait ressentir entre le rôle imparti aux collocations (rôle discutable) et les moyens d'analyse reconnus, accessibles (ce qui ne veut pas dire les seuls disponibles).

Un défrichement a eu lieu : des avancées sont encore pessibles, à condition que les disciplines en question dépassent leur sectarisme, que les praticiens cessent de croire en leur science infuse, que les linguistes soient à l'écoute des besoins et des problèmes de ceux et celles qui s'efforcent chaque jour de lever les malentendus.

YVES GAMBIER

Université de Turku, Truku, Finlande 